\title{
Attitudes and Barriers to Breastfeeding among Mothers in Princess Nourah Bint Abdulrahman University, Riyadh, Kingdom of Saudi Arabia
}

\author{
Razan Yasser Abulreesh, ${ }^{1}$ Ibtihaj Abdullah Alqahtani, ${ }^{1}$ Zainah Yahya Alshehri, ${ }^{1}$ \\ Maha Ali Alsubaie, ${ }^{1}$ Shatha Nasser Alburayh, ${ }^{1}$ Norah Mohammed Alzamil, ${ }^{2}$ \\ and Hayat Saleh Alzahrani ${ }^{2}$ \\ ${ }^{1}$ Faculty of Medicine, Princess Nourah Bint Abdulrahman University, Riyadh, Saudi Arabia \\ ${ }^{2}$ Family Medicine Department, Faculty of Medicine, Princess Nourah Bint Abdulrahman University, Riyadh, Saudi Arabia
}

Correspondence should be addressed to Hayat Saleh Alzahrani; hsaalzahrani@pnu.edu.sa

Received 27 February 2021; Revised 4 July 2021; Accepted 12 July 2021; Published 2 August 2021

Academic Editor: Jose Guilherme Cecatti

Copyright $\odot 2021$ Razan Yasser Abulreesh et al. This is an open access article distributed under the Creative Commons Attribution License, which permits unrestricted use, distribution, and reproduction in any medium, provided the original work is properly cited.

\begin{abstract}
Background. Breastfeeding provides unsurpassed natural nutrition to the newborn and infant. It has a nearly perfect mix of food elements and vitamins that infants need to grow up. Nonetheless, the tendency for breastfeeding remains below the expected levels. Objectives. To explore the attitudes and barriers to breastfeeding among mothers in Princess Nourah Abdulrahman University (PNU), Riyadh, Saudi Arabia. Methods. A cross-sectional study was conducted, from January to April 2019; 399 PNU students, employees, and faculty mothers aged 18 years and above with experience of childbirth and breastfeeding were included in the study using a predesigned validated questionnaire. The questionnaire consisted of four scales: sociodemographic, attitude toward breastfeeding, barriers to breastfeeding, and induced lactation knowledge. Results. The participants' mean age was $34.1 \pm 10.4$ years; most $(87.8 \%)$ were Saudi; $92.8 \%$ were married; $62 \%$ had a bachelor's degree; and $43 \%$ had "enough income." While $40 \%$ of the mothers reported $>6$ months "exclusive breastfeeding" for the first baby, only $34.8 \%$ did so for the last baby, and $54.5 \%$ did so for most of all babies altogether. The mothers' parity ranged between 1 birth and 4 births in $23.5 \%$ and $17.5 \%$ of the participants, respectively. An overall score of breastfeeding attitude averaged 59.6 \pm 7.3 . The tendency for scoring a negative attitude to breastfeeding was significantly reported $(p<0.5)$ among 127 (31.8\%) 31- to 40-year-old mothers; 153 (38.3\%) bachelor's degree holders; and 157 (39.3\%) employees $\left(\chi^{2}(4) 14.6, p=0.006 ; \chi^{2}(4) 10.4, p=0.034\right.$; and $\chi^{2}(4) 20.4, p<0.001$, respectively). "Mother's illness" was the most commonly (63\%) reported barrier to "not to breastfeed," followed by "work" (45.5\%) and "father not supporting breastfeeding" (14.8\%). Conclusions. An overall negative attitude toward breastfeeding among PNU mothers was noted. Barriers included mother's sickness and work. Efforts to minimize such negative attitudes and barriers among susceptible mothers are warranted.
\end{abstract}

\section{Introduction}

Breastfeeding provides unsurpassed natural nutrition to the newborn and infant [1]. It has a nearly perfect mix of food elements and vitamins that infants need to grow up. Nonetheless, the tendency for breastfeeding remains below the expected levels that is why the World Health Organization
(WHO) has set a goal of increasing the rate of exclusive breastfeeding to at least $50 \%$ by 2025 [2]. The benefits of breastfeeding in providing unique health and development opportunities for the infant are uncountable. Colostrum, which is secreted in the early hours following delivery, contains high levels of proteins, fats, and antibodies [3] together with leukocytes most of which are neutrophils and 
phagocytic macrophages that survive passage through the gastrointestinal system where they are absorbed and enforce the infant's immune response $[1,4]$.

Mature human breast milk then contains large quantities of secretory immunoglobulin A to line the respiratory and gastrointestinal mucosal membranes, rendering the infant four times less likely to develop diarrheal disease than formula-fed babies [5]. Such diarrheal disease is of shorter duration and is less severe than in formula-fed babies [6]. Likewise, breastfed infants exclusively for the first 6 months have a lower tendency for ear infections [7] and respiratory tract infections [8]. Compared to formula-fed infants, breastfed infants are more likely to gain the right amount of weight as they grow rather than become overweight children [9]. Further quality studies show that breastfeeding is associated with a significantly decreased incidence of type 2 diabetes and systolic blood pressure elevation later in life [10]. Evidently, infants who are breastfed exclusively for 6 months or longer compared with a shorter duration or not breastfeeding at all had a $20 \%$ lower risk for childhood leukemia [11].

The early feeding interactions, physical closeness, skinto-skin touching, and eye contact between mother and infant enhance maternal-infant bonding and result in more positive feeding experience and produce greater maternal sensitivity and responsiveness to infant needs [12].

The production of prolactin and oxytocin during breastfeeding is associated with lower levels of maternal stress and enhanced bonding [13]. On contrary, early cessation of breastfeeding or not breastfeeding at all has been linked to an increased risk of maternal postpartum depression [14]. Lactating women have been found at a lower risk for developing premenopausal breast and ovarian cancer [15]. On the child's part, too, the cognitive development is significantly enhanced because of the rich vital nutrients in breast milk, such as essential fatty acids, vitamins, minerals, and amino acids [16]. Language development [17] and overall neurological development are largely enhanced [13, 18]. For all these reasons, the WHO recommends that breastfeeding be initiated within one hour of birth that it continues with no other foods or liquids for the first six months of life and continued with other age-appropriate foods until at least 24 months of age [19].

Attitudes and confidence among women can predict the duration of exclusive breastfeeding; the longer the duration of breastfeeding, the more the advantages for both mother and child. On the other hand, barriers may be linked to problems related to either the mother, the infant, or the breastfeeding technique $[20,21]$. For instance, breast and nipple diseases as well as lactation complications, lack of selfefficacy, and limited social support may prevent mothers from lactating. Embarrassment toward breastfeeding in public together with an unsupportive childcare environment is a common cause of unfavorable breastfeeding practices [22, 23]. Maternal fatigue, stress, and other emotional problems are common health factors hindering exclusive breastfeeding. Mother's work particularly plays an important role in breastfeeding attitude. For instance, women planning to work full-time postpartum were less likely to initiate breastfeeding than women who planned to work part-time, and women were more likely to cease breastfeeding the first month prior or subsequent to returning to work [24]. Breastfeeding initiation and duration are reportedly lower among low-income women and women with lower education levels [25]. On the infant's part, being physically compromised or sustaining systemic illnesses interfere with the baby's ability to breastfeed successfully [20]. As a result of these and other problems, failure of initiation or discontinuity of breastfeeding is likely to occur.

In Saudi Arabia, there has been reluctance in practicing breastfeeding [26]; for example, only around 33\% of mothers exclusively breastfeed their infants for the first four months [3]. Mixing formula with breastfeeding, instead, is a popular infant feeding choice in Saudi Arabia [26, 27]. To date, there is no national database on breastfeeding in Saudi Arabia [26]. Furthermore, there is little research about barriers to breastfeeding in the country. This study explored attitudes toward breastfeeding and associated barriers and evaluated the knowledge about induction of lactation among mothers in PNU in Riyadh.

\section{Methods}

2.1. Study Design, Setting, and Study Population. A crosssectional design was selected for the study. The study was conducted in PNU in Riyadh, the capital of the Kingdom of Saudi Arabia (KSA), during the period between January 2019 and April 2019. Undergraduate students, employees, and faculty mothers aged 18 years and above with experience of childbirth and breastfeeding were included in the study. No PNU mother would be excluded from the study because of nationality, current marital status, occupation, or underlying health condition.

2.2. Study Sampling. Epi Info version 3 was used to calculate sample size " $n$," using sample size for "frequency in a proportion" formula ( $n=\mathrm{DEFF}^{*} N p$ $(1-p)) /\left(\left(d^{2} / Z^{2}{ }_{1-\alpha / 2}(N-1)+p^{*}(1-p)\right)\right)$, where population size (for finite population correction factor $(\mathrm{fpc}))(N)=$ 250,000; $p=$ hypothesized percentage frequency of outcome factor in the population $=50 \% \pm 5$ (to get maximum “ $n$ "); $d=$ confidence limits as percentage of 100 (absolute $+/-\%)=5 \%$; and design effect (DEFF) (for cluster surveys) $=1$ [28]. Our " $n$ " equated 384 , which was raised up to 412 to compensate for withdrawals and invalid responses. All mothers who agreed to participate were included in the study until the required sample size has been collected. The nature of the study population frame, like all were confined to PNU mothers, was not in favor of randomization; instead, a rather convenience sampling technique in recruiting the study participants' sample was adopted.

A validated self-administered questionnaire was developed to collect study data. The questionnaire consisted of four scales: sociodemographic, attitude toward breastfeeding, barriers to breastfeeding, and induced lactation knowledge. Sociodemographic scale included items (questions) relevant to breastfeeding, such as maternal age (years), 
nationality, marital status, level of education, occupation, living, income (all sources, all family members), payment, and number of live births. The breastfeeding attitude scale included items modified from the "Iowa infant feeding attitude scale" (IIFAS) [29]; IIFAS demonstrates high validity and reliability [30] and is widely utilized in the domain of measuring maternal attitudes toward infant feeding methods. The IIFAS consists of 17 items: 9 to reflect the positive attitudes to breastfeeding and 8 to reflect the negative ones (in favor of formula feeding). Items are scored on 5-point Likert-type scales, such as "strongly disagree," "disagree," "neutral," "agree," and "strongly agree". Items that favor breastfeeding are scored positively, so that "strongly agree" is given a maximum score of 5 , and a "strongly disagree" is given the least score of 1 . Items in favor of formula feeding are scored vice versa. An overall attitude score is computed via the equal-weighted sum of responses to all items, so that the total score ranges from 17 to 85 , with higher scores indicating positive attitudes to breastfeeding. Three main cutoff scores were set for our IIFAS: 17-60, $61-75$, and 76-85, delineating negative, neutral, and positive attitudes to breastfeeding, respectively [31, 32].

An Arabic scale translated from the original English IIFAS version was prepared, with utmost attention to ensure proper retention of the content and semantic and technical equivalence of this version. In order to ensure that the scale measures the same words and concepts, back translation of the Arabic scale as a second language to the English version as the first language was carried out. In developing the scale on barriers to breastfeeding, the investigators first examined a body of literature on relevant topics [22,33-36].

No standardized questionnaire with barriers to breastfeeding was available to advocate in the current study; therefore, we wanted to set items to address concepts pertinent to barriers toward breastfeeding, as concluded from the reviewed literature studies. For instance, principal "barriers" domains included: health status; breast condition, milk production, and impact of breastfeeding on breast shape; workplace and physical environment; cultural concerns, antenatal support, and postpartum support; emotional perception; and baby health issues. Accordingly, items identifying these concepts, including factors that encourage or discourage breastfeeding, have been phrased. Given that sensitive perception by many women to the inquiry about breastfeeding, anonymous inquiries were considered to allow mothers to answer more honestly and in a fast, efficient manner. Where appropriate, questions offered multiple-choice responses, so as the respondent could check more than one response [22].

Lastly, a scale on induction of lactation to integrate into the final questionnaire was also administered. Five items were introduced to identify the participants' opinion about relevant concepts, such as the possibility of inducing breast milk production, knowledge of ways to induce lactation, whether unmarried women are able to induce lactation, the possibility of reinducing lactation in postmenopausal women, and the possibility that woman who had never previously lactated can lactate successfully [37]. The final questionnaire was given to three consultants in the field: a pediatrician, a nutritionist, and a community health research scholar with an interest in maternal and child health promotion. The consultants were humbly asked to review and comprehensively evaluate the instrument in terms of construct and content validity. Refinement of the questionnaire draft was then done in response to the reviewers' notes and suggestions.

A pilot was conducted to assess the questionnaire's reliability. Twenty-five PNU mothers were given the questionnaire to respond (response-a). The same individuals were given the same questionnaire to readminister the questionnaire a week later (response-b). A panel of juries was selected to judge the responses; test-retest reliability was calculated to assess its temporal stability. Spearman's rho for each pair of items (response-a and response-b) was significant $(p<0.05)$ for the examined items, with moderate-strong correlation (rho 0.79-0.90). Modifications of the questionnaire items were carried out based on the pilot testing results. The piloted subjects were not included in the study. Most of the study items represent qualitative data, including nominal/dichotomous data, e.g., nationality, profession, barriers to breastfeeding (yes or no); discrete data, e.g., number of births; or ordinal data, e.g., education level, income stratum, number of times breastfed, and breastfeeding duration stratum.

2.3. Ethical Considerations. First, an application for ethical approval to conduct the study has been submitted to PNU Institutional Review Board (IRB) before commencing the study. The study has been approved by the IRB. Invited women were briefed of the study type and aim. Verbal consent was considered an agreement to participate in the study. A paper-and-pencil questionnaire form was given to participants on the PNU campus for their response. The questionnaire takes around 20 minutes to complete. Mothers were assured that their participation was voluntary and that they could opt out of the study at any time without giving reasons. They were further reassured of the confidentiality of the provided information and that only anonymous data would be disseminated. The returned questionnaires were coded, including data entered to a Microsoft program, with adequate backups until analyzed. Returned questionnaires with $\geq 80 \%$ valid answers would be entered into the analysis.

2.4. Statistical Analysis. Descriptive statistics in terms of count (\%) and the means \pm standard deviations (SD) or the median and interquartile ranges (IQR), where appropriate, would be used to describe the characteristics of the studied sample. In the inferential round of statistics, the strength of association between qualitative variables would be analyzed by the chi-square test of independence (or Fisher's exact test, where appropriate). Our alpha level to reject a true null hypothesis was $\alpha=0.05$, and the results with a $p$ values $<0.05$ were considered statistically significant.

\section{Results}

As in Table 1, 49.8\% of the mothers aged between 31 and 40, with mean age $34.1 \pm 10.4$ years (Table 1 , footnote). Also, $87.8 \%$ were Saudi; $92.8 \%$ were married; $62.5 \%$ had a 
TABLE 1: Distribution of participants by sociodemographic characteristics $(n=400)$.

\begin{tabular}{|c|c|c|}
\hline Category & Subcategory & $n(\%)$ \\
\hline \multirow{3}{*}{ Age (years) ${ }^{*}$} & $20-30$ & $118(29.5)$ \\
\hline & $31-40$ & $199(49.8)$ \\
\hline & $>40$ & $83(20.8)$ \\
\hline \multirow{2}{*}{ Nationality } & Saudi & $351(87.8)$ \\
\hline & Non-Saudi & $49(12.2)$ \\
\hline \multirow{2}{*}{ Marital status } & Married & $371(92.8)$ \\
\hline & Widow/divorced & $29(7.2)$ \\
\hline \multirow{3}{*}{ Level of education } & Before college & $67(16.8)$ \\
\hline & Bachelor's degree & $250(62.5)$ \\
\hline & Postgraduate & $83(20.8)$ \\
\hline \multirow{3}{*}{ Profession } & Student & $82(20.5)$ \\
\hline & Employee & $247(61.8)$ \\
\hline & Faculty member & $71(17.8)$ \\
\hline \multirow{4}{*}{ Living } & Own house & $162(40.5)$ \\
\hline & Rent & $148(37.0)$ \\
\hline & Large house & $45(11.2)$ \\
\hline & Workplace & $45(11.2)$ \\
\hline \multirow{5}{*}{ Income (all sources, all family members) } & Not enough but I borrow & $20(5.0)$ \\
\hline & Enough & $172(43.0)$ \\
\hline & Not enough & $62(15.5)$ \\
\hline & Enough and save & $97(24.2)$ \\
\hline & I don't want to answer & $49(12.2)$ \\
\hline \multirow{3}{*}{ Payment } & Governmental & $208(52.0)$ \\
\hline & Private & $103(25.8)$ \\
\hline & Health insurance & $89(22.0)$ \\
\hline \multirow{5}{*}{ Number of births } & One time & $94(23.5)$ \\
\hline & Two times & $90(22.5)$ \\
\hline & Three times & $74(18.5)$ \\
\hline & Four times & $70(17.5)$ \\
\hline & $>$ Four times & $72(18.8)$ \\
\hline
\end{tabular}

${ }^{*}$ Mean age $=34.1 \pm 10.4$.

bachelor's degree; and most (61.8\%) were employees. Livingwise, $40.5 \%$ of the mothers owned their house, and also, most of them $(43.0 \%)$ had enough income. Parity-wise, the frequency of having either one or two babies was almost equal $(23.5 \%$ and 22.5 , respectively) (Table 1$)$.

The first baby tended to be exclusively breastfed for $>6$ months more frequently (40\%) than the other 2 breastfeeding durations (36\% for 1-5 months and $24 \%$ for $<1$ month) (Table 2). In contrast, the last baby was breastfed more frequently for $<1$ month, compared with the other 2 breastfeeding durations $(34.8 \%$ for $>6$ months and $24.2 \%$ for $<1$ month). As in the first baby trend, most of all babies were breastfeed most reportedly (54.5\%) for $>6$ months, $30 \%$ for 1-5 months, and least reportedly (15.5\%) for $<1$ month (Table 2).

The majority of the mothers reported that sickness was the largest barrier that prevented them from breastfeeding their baby (63\%), and the second barrier was work $(46.5 \%)$. Lack of encouragement from fathers was found to be the least common barrier toward breastfeeding (14.8\%) (Table 3).

Table 4 displays attitudinal responses toward breastfeeding questions. Mothers were generally negative toward breastfeeding (mean total score $=59.6 \pm 7.3$ ). The mean score
TABLE 2: Distribution of the study participants by the duration of exclusive breastfeeding $(n=400)$.

\begin{tabular}{lcc}
\hline Child order & Breastfeeding duration & $n(\%)$ \\
\hline \multirow{3}{*}{ First baby } & Less than one month & $96(24.0)$ \\
& $1-5$ months & $144(36.0)$ \\
& More than 6 months & $160(40.0)$ \\
\hline \multirow{3}{*}{ Last baby } & Less than one month & $164(41.0)$ \\
& $1-5$ months & $97(24.2)$ \\
& More than 6 months & $139(34.8)$ \\
Most of all babies & Less than one month & $62(15.5)$ \\
& $1-5$ months & $120(30.0)$ \\
& More than 6 months & $218(54.5)$ \\
\hline
\end{tabular}

was not significantly different from the cutoff limit of 60 for negative attitudes against breastfeeding $(t$ df 399) $=-1.37$; $p=0.17$ ) (Table 4 footnotes). The majority of responses on most of the 8 questions that reflect negative attitudes to breastfeeding were unfavorable. For instance, 117 (29.2\%) agreed that the "benefits of breast milk last only until weaning" (question 1 , mean score $=2.9 \pm 1.4$ ) (Table 4). Likewise, as many as $183(45.8 \%)$ agreed that "formula feeding was the better choice to plan to go out to work" (question 6, mean score $=2.4 \pm 1.1$ ); $93(23.2 \%$ ) agreed that "women should not breastfeed in places" (question 8, mean 
TABLE 3: Distribution of the study group by barriers to breastfeeding $(n=400)$.

\begin{tabular}{lcc}
\hline$\#$ & Barrier & $n(\%)$ \\
\hline 1 & Sick & $252(63.0)$ \\
2 & Work & $186(46.5)$ \\
3 & Tired & $174(43.5)$ \\
4 & Taking contraceptives & $165(41.2)$ \\
5 & Disease could transfer to the baby through breastfeeding & $164(41.0)$ \\
6 & Too busy to breastfeed the baby & $163(40.8)$ \\
7 & Embarrassed from lactation in public places & $156(39.0)$ \\
8 & Perception of insufficient milk production \\
9 & Pain presents an obstacle to breastfeeding & $129(32.2)$ \\
10 & Depressed because my child refused breastfeeding \\
11 & Poor prenatal and postpartum support & $122(30.5)$ \\
12 & Unpleasant odor in the nursing mom & $114(28.5)$ \\
13 & Housekeeper availability encourages me to provide bottle-feeding & $105(26.2)$ \\
14 & I don't have enough knowledge & $81(20.2)$ \\
15 & Fear of distorted breast shape by breastfeeding & $79(19.8)$ \\
16 & Father does not encourage breastfeeding & $67(16.8)$ \\
\hline
\end{tabular}

TABLE 4: Itemized scoring of the mothers' attitudes to breastfeeding.

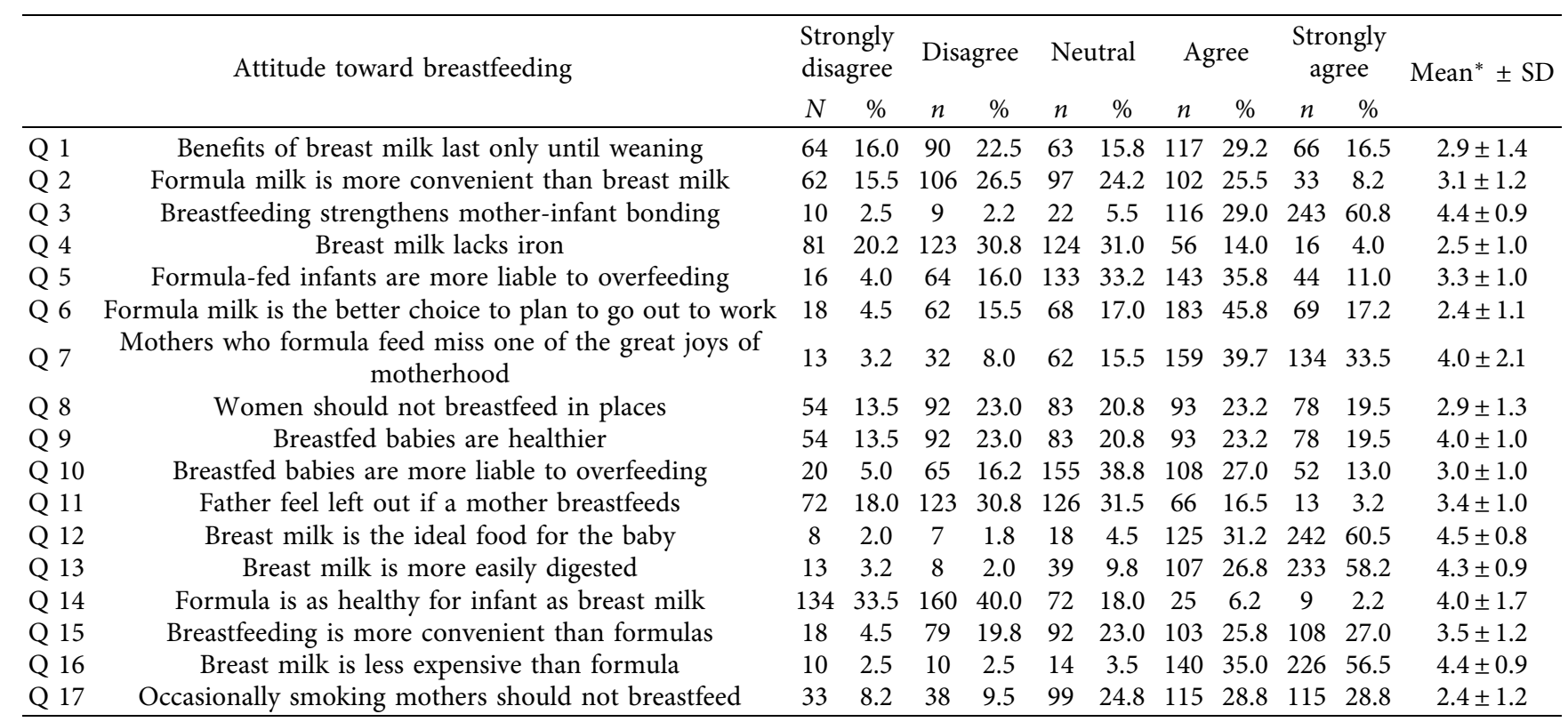

${ }^{*}$ Participants' mean score $=59.6 \pm 7.3$; one sample $t$-test: $t(\mathrm{df} 399)=-1.37 ; p=0.17$.

score $=2.9 \pm 1.3)$; and $108(27.0 \%)$ believed that "breastfed babies are more liable to overfeeding" (question 10, mean score $=3 \pm 1)$.

In Table 5, three main attitudinal categories to breastfeeding are shown: negative, neutral, and positive, in relationship with ten sociodemographic characteristics examined. Only four (age, level of education, profession, and healthcare payment method) characteristics showed a significant influence upon breastfeeding $\left(\chi^{2} \quad(4)=14.6\right.$, $p=0.006 ; \chi^{2}(4)=10.4, p=0.034 ; \chi^{2}(4)=20.4, p<0.001$; and $\chi^{2}(4)=12.2, p=0.016$, respectively). First, an overall $223(55.8 \%)$ participants scored a negative attitude, 171 (42.7\%) participants were neutral, and only $6(1.5 \%)$ were positive to breastfeeding (Table 5 footnote). In 3 out of the 4 significant analyses, the most frequently occurring observation was that for the negative attitude and other attitudes in the same sociodemographic comparison, e.g., 127 (63.8\% of row and $31.8 \%$ of total 400 ) of 31-40 years old. Also, 153 (51.2\% of row and $38.3 \%$ of total) of bachelor holders and 157 (63.6\% of row and $39.3 \%$ of total) of employees expressed a negative attitude to breastfeeding. On the other hand, postgraduate educated mothers reacted more favorably to breastfeeding that $45 / 83(54.2 \%)$ were neutral, while $36 / 83$ (43.4\%) were negative to breastfeeding. Likewise, $43 /$ $71(60.6 \%)$ of faculty members were neutral to breastfeeding, compared to $86 / 247$ (3 4.8\%) of employees and $42 / 82$ $(51.2 \%)$ of students. In the health care payment type comparison, 103 (49.5\% of row and $25.8 \%$ of total) of the governmentally insured were neutral, followed by 100 ( $48.1 \%$ of row and $25 \%$ of total) of the same insurance category who were negative to breastfeeding. Income, the number of births, and the number of times mothers ever 
TABLE 5: Distribution of breastfeeding attitude by sociodemographic characteristics: age, nationality, marital status, education, profession, living, payment, and income.

\begin{tabular}{|c|c|c|c|c|c|c|c|c|c|c|c|}
\hline \multirow{3}{*}{ Characteristic } & \multirow{3}{*}{ Category } & \multicolumn{9}{|c|}{ Attitude to breastfeeding* } & \multirow{3}{*}{$p$ value } \\
\hline & & \multicolumn{2}{|c|}{$\begin{array}{c}\text { Negative } \\
(17-60)\end{array}$} & \multicolumn{2}{|c|}{$\begin{array}{l}\text { Neutral } \\
(61-75)\end{array}$} & \multicolumn{2}{|c|}{$\begin{array}{l}\text { Positive } \\
(76-88)\end{array}$} & \multicolumn{2}{|c|}{ Total } & \multirow[t]{2}{*}{ Test statistic } & \\
\hline & & $N$ & $\%$ & $n$ & $\%$ & $n$ & $\%$ & $n$ & $\%$ & & \\
\hline \multirow{3}{*}{ Age (years) } & $20-30$ & 63 & 53.4 & 53 & 44.9 & 2 & 1.7 & 118 & 100 & \multirow{3}{*}{$\chi^{2}(4)=14.6$} & \multirow{3}{*}{0.006} \\
\hline & $31-40$ & 127 & 63.8 & 69 & 34.7 & 3 & 1.5 & 199 & 100 & & \\
\hline & More than 40 & 33 & 39.8 & 49 & 59.0 & 1 & 1.2 & 83 & 100 & & \\
\hline \multirow{2}{*}{ Nationality } & Saudi & 199 & 56.7 & 147 & 41.9 & 5 & 1.4 & 351 & 100 & \multirow{2}{*}{$\chi^{2}(1)=1.1$} & \multirow{2}{*}{0.58} \\
\hline & Non-Saudi & 24 & 49.0 & 24 & 49.0 & 1 & 2.0 & 49 & 100 & & \\
\hline \multirow{2}{*}{ Marital status } & Married & 206 & 55.5 & 159 & 42.9 & 6 & 1.6 & 371 & 100 & \multirow{2}{*}{$\chi^{2}(1)=0.53$} & \multirow{2}{*}{0.77} \\
\hline & Widow/divorced & 17 & 58.6 & 12 & 41.4 & 0 & 0.0 & 29 & 100 & & \\
\hline \multirow{3}{*}{ Level of education } & Before college & 34 & 50.7 & 33 & 49.3 & 0 & 0.0 & 67 & 100 & \multirow{3}{*}{$\chi^{2}(4)=10.4$} & \multirow{3}{*}{0.034} \\
\hline & Bachelor & 153 & 61.2 & 93 & 37.2 & 4 & 1.6 & 250 & 100 & & \\
\hline & Postgraduate & 36 & 43.4 & 45 & 54.2 & 2 & 2.4 & 83 & 100 & & \\
\hline \multirow{3}{*}{ Profession } & Student & 40 & 48.8 & 42 & 51.2 & 0 & 0.0 & 82 & 100 & \multirow{3}{*}{$\chi^{2}(4)=20.4$} & \multirow{3}{*}{$<0.001$} \\
\hline & Employee & 157 & 63.6 & 86 & 34.8 & 4 & 1.6 & 247 & 100 & & \\
\hline & Faculty member & 26 & 36.6 & 43 & 60.6 & 2 & 2.8 & 71 & 100 & & \\
\hline & Own house & 92 & 56.8 & 67 & 41.4 & 3 & 1.9 & 162 & 100 & & \\
\hline & Rent & 90 & 60.8 & 57 & 38.5 & 1 & 0.7 & 148 & 100 & & \\
\hline Living & Large house & 22 & 48.9 & 22 & 48.9 & 1 & 2.2 & 45 & 100 & $\chi^{2}(6)=6.5$ & 0.37 \\
\hline & Workplace & 19 & 42.2 & 25 & 55.6 & 1 & 2.2 & 45 & 100 & & \\
\hline & Governmental & 100 & 48.1 & 103 & 49.5 & 5 & 2.4 & 208 & 100 & & \\
\hline Healthcare payment & Private & 68 & 66.0 & 35 & 34.0 & 0 & 0.0 & 103 & 100 & $\chi^{2}(4)=12.2$ & 0.016 \\
\hline & Health insurance & 55 & 61.8 & 33 & 37.1 & 1 & 1.1 & 89 & 100 & & \\
\hline & Not enough; borrow & 9 & 45.0 & 11 & 55.0 & 0 & 0.0 & 20 & 100 & & \\
\hline & Enough & 102 & 59.3 & 67 & 39.0 & 3 & 1.7 & 172 & 100 & & \\
\hline Income & Not enough & 35 & 56.5 & 26 & 41.9 & 1 & 1.6 & 62 & 100 & $\chi^{2}(8)=4.4$ & 0.82 \\
\hline & Enough and save & 49 & 50.5 & 46 & 47.4 & 2 & 2.1 & 97 & 100 & & \\
\hline & Don't answer & 28 & 57.1 & 21 & 42.9 & 0 & 0 & 49 & 100 & & \\
\hline & One time & 50 & 53.2 & 43 & 45.7 & 1 & 1.1 & 94 & 100 & & \\
\hline & Two times & 52 & 57.8 & 36 & 40.0 & 2 & 2.2 & 90 & 100 & & \\
\hline Number of births & Three times & 44 & 59.5 & 28 & 37.8 & 2 & 2.7 & 74 & 100 & $\chi^{2}(8)=6.2$ & 0.62 \\
\hline & Four times & 43 & 61.4 & 27 & 38.6 & 0 & 0 & 70 & 100 & & \\
\hline & $>$ Four times & 34 & 47.2 & 37 & 51.4 & 1 & 1.4 & 72 & 100 & & \\
\hline & One baby & 57 & 52.3 & 52 & 47.7 & 0 & 0 & 109 & 100 & & \\
\hline & Two babies & 66 & 58.4 & 45 & 39.8 & 2 & 1.8 & 113 & 100 & & \\
\hline Number of breastfed babies & Three babies & 45 & 60.8 & 25 & 33.8 & 4 & 5.4 & 74 & 100 & $\chi^{2}(8)=15.2$ & 0.0 .55 \\
\hline & Four babies & 33 & 56.9 & 25 & 43.1 & 0 & 0 & 58 & 100 & & \\
\hline & $>$ Four babies & 22 & 47.8 & 24 & 52.2 & 0 & 0 & 46 & 100 & & \\
\hline
\end{tabular}

*223 (55.8\%) scored a negative attitude; $171(\% 42.7)$ were neutral; and $6(1.5 \%)$ scored a positive attitude.

breastfed did not influence the attitude to breastfeeding $(p<0.05$ all analyses).

A total of $73.5 \%$ of participants agreed with the concept of "induced lactation" (Table 6). The "most known way to increase milk production" was "drugs and herbal remedies". Otherwise, $14.2 \%$ of the participants were uncertain about "the ability of premenopausal women to induce lactation. Only $9 \%$ of the participants were against reinducing lactation in nonlactating females (Table 6 footnote).

\section{Discussion}

Most infants today still do not receive the full benefits of breastfeeding, leaving millions at unnecessary risk of illness and death, and most health workers lack the skills needed to help mothers improve their feeding practices [38]. The problem involves a multitude of attitudinal issues besides issues that pose barriers to exclusive breastfeeding. Most of our participants' responses to those questions addressing negative attitudes to breastfeeding were unfavorable. As such, $55.8 \%$ of PNU mothers responded negatively, and $42.7 \%$ were neutral to breastfeeding, all with a mean score (59.6) that did not significantly differ from the study instrument's cutoff score of 60 . In Riyadh, the study by Saied et al. [3] showed that the majority (89.4\%) of mothers had a neutral attitude toward breastfeeding. The more likelihood of a negative attitude to breastfeeding in our study may be attributed to the situation that most $(61.8 \%)$ of our participants were working mothers, while $63.1 \%$ of Riyadh's mothers were housewives. 
TABLE 6: The participants' attitude toward induced lactation concept.

\begin{tabular}{|c|c|c|c|}
\hline Group* & Agree $n(\%)$ & Disagree $n(\%)$ & Total $n(\%)$ \\
\hline Lactation can be indicted in married women & $294(73.5)$ & $106(26.5)$ & $400(100)$ \\
\hline Lactation can be indicted in premenopausal women & $57(14.2)$ & $343(85.8)$ & $400(400)$ \\
\hline
\end{tabular}

*All participants' attitudes to reinducing lactation in postmenopausal women: $91 \%$ agree and $9 \%$ disagree.

Among our findings, age less than 40 was more likely met with a negative attitude to breastfeeding. Comparably, Laanterä et al. found that women below 27 did not tend to initiate breastfeeding [39]. In this work, Saudi mothers did not differ in their attitude to breastfeeding with peers of other nationalities, for example, contrary to what had been found when Saudis were compared with Egyptian peers, where only $14 \%$ of Saudi mothers reacted negatively to exclusive breastfeeding versus $65 \%$ of Egyptian mothers who behaved otherwise [26].

Mothers who were living in their workplace residency had a more positive attitude $(53.3 \%)$ due to the close proximity, a finding which was also in line with that reported by other studies [40].

Pooled data analysis of a large population-based study from Nepal revealed that maternal education was consistently associated with a higher likelihood of early initiation of breastfeeding [41]. In our study, only postgraduate education and being academia were associated with a neutral attitude, rather than a negative attitude to breastfeeding.

The primary barrier preventing the initiation of breastfeeding among PNU mothers was sickness. Likewise, mothers' sickness was the second most commonly given reason for discontinuation of breastfeeding among mothers in Riyadh, KSA [42]. Work commitment was the second barrier in our study (46.5\%). In fact, the mother's work has been consistently a significant barrier to all aspects of breastfeeding practice. For instance, Kimbro [24] found that the timings a lactating mother quits breastfeeding and that when she returns to work were closely linked, and that mothers in administrative and manual positions tended to quit earlier than other women. Dunn et al. [22] reported that breastfeeding duration significantly correlated with the mother's employment status and that among women who breastfed for 6 months or longer, only 15\% were employed full-time, $30 \%$ worked part-time, and $55 \%$ were not committed to work. Likewise, Lauer et al. [43] found that women in specific service-oriented industries (accommodation and retail) reported the lowest rates of breastfeeding initiation and workplace supports for breastfeeding and pumping.

In our study, $39 \%$ of mothers were embarrassed about breastfeeding their babies in public places. In a study to reveal barriers to breastfeeding in Kuwait [44], most mothers reported feeling embarrassed to practice breastfeeding in public, e.g., $85.5 \%$ felt embarrassed to pump their breasts at work, $74.9 \%$ felt shy about breastfeeding outside the home, and $60.7 \%$ feared that people would see their breasts. Likewise, a study in Mississippi, USA, showed similar results of a sense of embarrassment, specifically about pumping breasts at school or work, breastfeeding outside their homes, and breastfeeding in front of family members [45]. In this work, too, mothers who indicated that the father does not encourage breastfeeding accounted for the least proportion among barriers to breastfeeding (14.8\%). In Kuwait, too, among the members of social support, the more frequently mentioned persons were the husbands (84.7\%) [44]. In the Mississippi study [45], time and social constraints presented barriers to fewer women than the embarrassment issues. In the present study, the embarrassment barrier was the most common, and lack of social support was perceived as the least common barrier.

Medical advances now developed techniques facilitating induced lactation and allowing nulliparous mothers the ability to breastfeed their children [45]. The present study demonstrated that only $9 \%$ and $14 \%$ of participants agreed that women can breastfeed at any age and that lactation can be induced in nonlactating women, respectively, indicating a knowledge gap about induced lactation. These findings were similar to what have been found in Malaysia, where also herbal medication was the most commonly known method to increase breast milk production [46].

4.1. Strengths and Limitations. The evidence-based data collecting instrument enabled identifying and measuring aspects of breastfeeding behavior and correlates the study aimed to examine, ensuring a level of validity of the achieved data. The relatively large sample size as well as the high testretest and reproducibility of the developed questionnaire assure adequate generalizability of the obtained results in the general population setting.

The chi-square test may not show the optimal results in consideration of the low number of subjects, for example, the marital status and nationality (Table 5).

On the other hand, selecting the study sample in professional strata as exactly proportionate as those of PNU mothers' population would have been impractical; the reason why utilizing a convenience sampling technique in recruiting the calculated sample size was an alternative.

In addition, information regarding the benefits of breastfeeding was provided along with participants' questions were discussed and answered.

\section{Conclusions}

This research demonstrates a predominance of a negative attitude toward breastfeeding among PNU mothers. Significant relationships are noted between this attitude and age, nationality, education, profession, payment, and living. Older, more highly educated, and non-Saudi mothers had more positive attitudes toward breastfeeding. In contrast, the relationship between income, number of births, and number of times breastfeeding was less significant. Mother's illness and work represented barriers with the highest percentages 
among reasons preventing breastfeeding, and several other barriers are also noted. There is good information about how to increase the production of milk, yet information about the induction of lactation was limited.

5.1. Recommendations. With respect to the obtained findings that were less likely in favor of breastfeeding and the associated determinants, particular recommendations include: (a) providing health education programs in student curricula, (b) support the creation of a breastfeedingfriendly work environment, and (c) conducting awareness campaigns about induction of lactation. Further research to provide guidance regarding inducing lactation in Saudi Arabia is suggested.

\section{Data Availability}

All data associated with this study are present in the paper.

\section{Ethical Approval}

The study was approved by the Institutional Review Board (IRB)/Research Ethics Committee (REC) of Princess Nourah Bint Abdulrahman University (IRB/Ethical Approval Log Number: 19-0007).

\section{Consent}

Written and oral informed consent was obtained from all individual participants included in the study. Additional informed consent was obtained from all individual participants for whom identifying information is included in this manuscript.

\section{Conflicts of Interest}

The authors of the manuscript declare that there are no conflicts of interest.

\section{Authors' Contributions}

All authors read and approved the final manuscript.

\section{Acknowledgments}

This research was funded by the Deanship of Scientific Research at Princess Nourah Bint Abdulrahman University, Riyadh, Saudi Arabia, through the Fast-track Research funding Program.

\section{References}

[1] K. M. Jackson and A. Nazar, "Breastfeeding, the immune response, and long-term health," The Journal of the American Osteopathic Association, vol. 106, pp. 203-207, 2006.

[2] World Health Organization (WHO), "Comprehensive implementation plan on maternal, infant and young child nutrition," 2014, https://www.who.int/nutrition/publications/ CIP_document/en/.

[3] H. Saied, A. Mohamed, A. Suliman, and W. Al Anazi, "Breastfeeding knowledge, attitude and barriers among Saudi women in Riyadh," Journal of Natural Sciences Research, vol. 3, no. 12, pp. 6-13, 2013.

[4] A. E. Wold and I. Adlerberth, "Does breastfeeding affect the infant's immune responsiveness?" Acta Paediatrica (Oslo, Norway: 1992), vol. 87, pp. 19-22, 1998.

[5] M. A. Quigley, P. Cumberland, J. Cowden, and L. Rodrigues, "How protective is breast feeding against diarrhoeal disease in infants in 1990s England? A case-control study," Archives of Disease in Childhood, vol. 91, no. 3, pp. 245-250, 2006.

[6] L. M. Lamberti, C. L. F. Walker, A. Noiman, C. Victora, and R. E. Black, "Breastfeeding and the risk for diarrhoea morbidity and mortality," BMC Pub Health, vol. 11, no. 3, p. S15, 2013.

[7] G. Bowatte, R. Tham, K. Allen et al., "Breastfeeding and childhood acute otitis media: a systematic review and metaanalysis," Acta Paediatrica, vol. 104, pp. 85-95, 2015.

[8] I. Tromp, J. Kiefte-de Jong, H. Raat et al., "Breastfeeding and the risk of respiratory tract infections after infancy: the Generation R Study," PLoS One, vol. 12, no. 2, Article ID e0172763, 2017.

[9] M. Onis and A. Onyango, "The centers for disease control and prevention 2000 growth charts and the growth of breastfed infants," Acta Paediatrica, vol. 92, no. 4, pp. 413-419, 2003.

[10] B. L. Horta, C. Loret de Mola, and C. G. Victora, "Long-term consequences of breastfeeding on cholesterol, obesity, systolic blood pressure and type 2 diabetes: a systematic review and meta-analysis," Acta Paediatrica, vol. 104, pp. 30-37, 2015.

[11] E. L. Amitay and L. Keinan-Boker, "Breastfeeding and childhood leukemia incidence," JAMA Pediatrics, vol. 169, no. 6, Article ID e151025, 2015.

[12] S. J. DeWitt, J. W. Sparks, P. B. Swank, K. Smith, S. E. Denson, and S. H. Landry, "Physical growth of low birthweight infants in the first year of life: impact of maternal behaviors," Early Human Development, vol. 47, no. 1, pp. 19-34, 1997.

[13] R. Uauy and I. de Andraca, "Human milk and breast feeding for optimal mental development," The Journal of Nutrition, vol. 125, pp. 2278S-2280S, 1995.

[14] S. Ip, M. Chung, G. Raman et al., "Breastfeeding and maternal and infant health outcomes in developed countries," Evidence report/technology assessment, vol. 153, pp. 1-186, 2007.

[15] A. Ahmed and S. R. El Guindy, "Breastfeeding knowledge and attitudes among Egyptian baccalaureate students," International Nursing Review, vol. 58, no. 3, pp. 372-378, 2011.

[16] P. Quinn, M. O’Callaghan, G. Williams, J. Najman, M. Andersen, and W. Bor, "The effect of breastfeeding on child development at 5 years: a cohort study," Journal of Paediatrics and Child Health, vol. 37, no. 5, pp. 465-469, 2001.

[17] A. J. O. Whitehouse, M. Robinson, J. Li, and W. H. Oddy, "Duration of breast feeding and language ability in middle childhood," Paediatric and Perinatal Epidemiology, vol. 25, no. 1, pp. 44-52, 2011.

[18] R. Feldman and A. I. Eidelman, "Direct and indirect effects of breast milk on the neurobehavioral and cognitive development of premature infants," Developmental Psychobiology, vol. 43, no. 2, pp. 109-119, 2003.

[19] World health organization (WHO), "Global breastfeeding scorecard, 2017. Tracking progress for breastfeeding policies and programmes," 2017, https://www.who.int/nutrition/ publications/infantfeeding/global-bf-scorecard-2017.pdf.

[20] J. Callen, J. Pinelli, S. Atkinson, and S. Saigal, "Qualitative analysis of barriers to breastfeeding in very-low-birth weight infants in the hospital and post discharge," Advances in Neonatal Care, vol. 5, no. 2, pp. 93-103, 2005.

[21] K. A. Dearden, L. N. Quan, and M. Do, "Work outside the home is the primary barrier to exclusive breast feeding in rural 
Vietnam: insights from mothers who exclusively breast fed and worked," Food and Nutrition Bulletin, vol. 23, no. 4, pp. 101-108, 2002.

[22] R. L. Dunn, K. A. Kalich, R. Fedrizzi, and S. Phillips, "Barriers and contributors to breastfeeding in WIC mothers: a social ecological perspective," Breastfeeding Medicine, vol. 10, no. 10, pp. 493-501, 2015.

[23] A. V. Holmes, N. P. Chin, J. Kaczorowski, and C. R. Howard, "A barrier to exclusive breastfeeding for WIC enrollees: limited use of exclusive breastfeeding food package for mothers," Breastfeeding Medicine, vol. 4, no. 1, pp. 25-30, 2009.

[24] R. T. Kimbro, "On-the-job moms: work and breastfeeding initiation and duration for a sample of low-income women," Maternal and Child Health Journal, vol. 10, no. 1, pp. 19-26, 2006.

[25] K. R. Mirkovic, C. G. Perrine, K. S. Scanlon, and L. M. Grummer-Strawn, "Maternity leave duration and fulltime/part-time work status are associated with US mothers' ability to meet breastfeeding intentions," Journal of Human Lactation, vol. 30, no. 4, pp. 416-419, 2014.

[26] H. M. Elsayed and L. A. Al-Dossary, "Exclusive breastfeeding, prevalence and maternal concerns: Saudi and Egyptian mothers," Journal of Education and Practice, vol. 7, pp. 5-11, 2016.

[27] M. S. Al-Jassir, B. M. El-Bashir, S. K. Moizuddin, and A. A. R. Abu-Nayan, "Infant feeding in Saudi Arabia: mothers' attitudes and practices," Eastern Mediterranean Health Journal, vol. 12, pp. 6-13, 2006, http://pesquisa.bvsalud.org/ portal/resource/es/mdl-17037217.

[28] A. G. Dean, K. M. Sullivan, and M. M. Soe, "Openepi: open source epidemiologic statistics for public health," 2013, http:// www.openepi.com/Menu/OE_Menu.htm\%20updated\%202013/ $04 / 06$.

[29] A. D. L. Mora, D. W. Russell, C. I. Dungy, M. Losch, and L. Dusdieker, "The Iowa infant feeding attitude scale: analysis of reliability and Validity," Journal of Applied Social Psychology, vol. 29, no. 11, pp. 2362-2380, 1999.

[30] I. Shaker, J. A. Scott, and M. Reid, "Infant feeding attitudes of expectant parents: breastfeeding and formula feeding," Journal of Advanced Nursing, vol. 45, no. 3, pp. 260-268, 2004.

[31] L. Charafeddine, H. Tamim, M. Soubra, A. de la Mora, M. Nabulsi, and Research and Advocacy Breastfeeding Team, "Validation of the Arabic version of the Iowa infant feeding attitude scale among Lebanese women," Journal of Human Lactation: Official Journal of International Lactation Consultant Association, vol. 32, no. 2, pp. 309-314, 2016.

[32] M. Abdulahi, A. Fretheim, A. Argaw, and J. H. Magnus, "Adaptation and validation of the Iowa infant feeding attitude scale and the breastfeeding knowledge questionnaire for use in an Ethiopian setting," International Breastfeeding Journal, vol. 15, no. 24, 2020.

[33] S. S. Hawkins, L. J. Griffiths, C. Dezateux, and C. Law, "Millennium cohort study child health group maternal employment and breast-feeding initiation: findings from the millennium cohort study," Paediatric and Perinatal Epidemiology, vol. 21, no. 3, pp. 242-247, 2007.

[34] M. L. Johnston and N. Esposito, "Barriers and facilitators for breastfeeding among working women in the United States," Journal of Obstetric, Gynecologic \& Neonatal Nursing, vol. 36, no. 1, pp. 9-20, 2007.

[35] R. K. Dagher, P. M. McGovern, J. D. Schold, and X. J. Randall, "Determinants of breastfeeding initiation and cessation among employed mothers: a prospective cohort study," BMC Pregnancy and Childbirth, vol. 16, no. 1, p. 194, 2016.

[36] I. C. Hedberg, "Barriers to breastfeeding in the WIC population," MCN: The American Journal of Maternal/Child Nursing, vol. 38, no. 4, pp. 244-249, 2013.

[37] K. Nemba, "Induced lactation: a study of 37 non-puerperal mothers," Journal of Tropical Pediatrics, vol. 40, no. 4, pp. 240-242, 1994.

[38] H. M. Shaheen, N. N. Hegazy, and S. A. Sakr, "The barriers to breastfeeding among women: a single-center experience," Menoufia Medical Journal, vol. 31, no. 3, pp. 855-861, 2018.

[39] S. Laanterä, T. Pölkki, A. Ekström, and A. M. Pietilä, "Breastfeeding attitudes of Finnish parents during pregnancy," BMC Pregnancy and Childbirth, vol. 10, no. 79, 2010.

[40] J. Chen, T. Xin, J. Gaoshan et al., "The association between work related factors and breastfeeding practices among Chinese working mothers: a mixed-method approach," International Breastfeeding Journal, vol. 14, no. 28, 2019.

[41] P. Acharya and V. Khanal, "The effect of mother's educational status on early initiation of breastfeeding: further analysis of three consecutive Nepal Demographic and Health Surveys," BMC Public Health, vol. 15, no. 1, p. 1069, 2015.

[42] Y. A. Alwelaie, E. A. Alsuhaibani, A. M. Al-Harthy, R. H. Radwan, R. G. Al-Mohammady, and A. M. Almutairi, "Breastfeeding knowledge and attitude among Saudi women in Central Saudi Arabia," Saudi Medical Journal, vol. 31, no. 2, pp. 193-198, 2010.

[43] E. Lauer, K. Armenti, M. Henning, and L. Sirois, "Identifying barriers and supports to breastfeeding in the workplace experienced by mothers in the New Hampshire special supplemental nutrition program for women, infants, and children utilizing the total worker health framework," International Journal of Environmental Research and Public Health, vol. 16, no. 4, p. 529, 2019.

[44] F. Al-Darweesh, R. Al-Hendyani, K. Al-Shatti et al., "Knowledge, intention, practice, and perceived barriers of breastfeeding among married working women in Kuwait," International Journal of Family Medicine, vol. 1, pp. 108-113, 2016.

[45] A. J. Khoury, A. K. Mitra, A. Hinton, C. Carothers, and H. Sheil, "An innovative video succeeds in addressing barriers to breastfeeding among low-income women," Journal of Human Lactation, vol. 18, no. 2, pp. 125-131, 2002.

[46] N. Che Abdul Rahim, Z. Sulaiman, Z. Sulaiman, and T. A. Tengku Ismail, "The availability of information on induced lactation in Malaysia," Malaysian Journal of Medical Sciences, vol. 24, no. 4, pp. 5-17, 2017. 